\title{
Acute femoral fracture does not compromise the outcome of total hip arthroplasty : 5 year results from a single center cohort study in $\mathbf{2 , 7 8 2}$ patients
}

\author{
Stefan B.T. Bolder, Elsa A. Spaans, Joost A.A.M. Van den Hout, Robert Wagenmakers, Koen L.M. KoenraAdt
}

From the Department of Orthopedic Surgery, Amphia Hospital, Breda, The Netherlands

National arthroplasty registries reveal a higher risk of revision for periprosthetic fracture and dislocation after total hip arthroplasty (THA) in patients with a femoral neck fracture compared to those with osteoarthritis (OA). Since these registries may contain confounding factors, we conducted a single center cohort study comparing survival and reason for failure between THA for an acute femoral neck fracture and $\mathrm{OA}$ using the same hip prosthesis after a minimum follow-up of 2 years.

We retrospectively analyzed 2782 patients who had undergone THA with an Accolade TMZF stem and a Trident cup between March 2009 and September 2014. Primary diagnosis before THA was osteoarthritis (OA group : $n=2610$ ) or acute femoral fracture (Fracture group : $n=172$ ). Patients in both groups were operated on by the same hip surgeons. Effect of diagnosis on THA survival was analyzed using Cox-regression analysis. Chi-square tests were used to illustrate the different reasons for revision between the groups.

Mean follow-up was 4.6 years (2-7.6). A total of 100 revisions were performed. The revision risk was comparable between the $\mathrm{OA}$ and Fracture group $(H R=1.04,95 \%$ CI :0.46-2.39). No differences were found between the $\mathrm{OA}$ and Fracture group with respect to the occurrence of periprosthetic fractures $(0.8 \%$ vs. $0.6 \%, p=0.71)$ and revisions for dislocation $(0.6 \%$ vs. $1.2 \%, p=0.38)$.

We found no difference in outcome or reason for revision between THA for OA or femoral neck fracture. Using an implant with a proven ODEP rating and having experienced hip surgeons carry out the procedures may be more important than the primary diagnosis.
Keywords : hip ; arthroplasty ; femoral neck fracture ; osteoarthritis.

\section{INTRODUCTION}

A growing number of acute femoral neck fractures are treated with total hip arthroplasty (THA) (1). Currently, $4.9 \%$ of the THAs are performed for acute femoral neck fracture (1). Many national hip registries have shown that a good outcome of THA in patients with an acute femoral neck fracture can be obtained, but when compared to primary THA in osteoarthritis (OA) patients, inferior outcomes in fracture patients were identified (14). Dislocations and periprosthetic fractures were more frequently implicated as a reason for failure in

Stefan B.T. Bolder ${ }^{1}, \mathrm{MD}, \mathrm{PhD}$,

Elsa A. Spaans ${ }^{1}, \mathrm{MD}$,

- Joost A.A.M. Van den Hout ${ }^{1}$, MD,

Robert Wagenmakers ${ }^{1}, \mathrm{MD} \mathrm{PhD}$,

- Koen L.M. Koenraadt ${ }^{2}$, PhD

1 Department of Orthopedic Surgery, Amphia Hospital, Breda, The Netherlands.

${ }^{2}$ Foundation for Orthopedic Research Care and Education (FORCE), Amphia Hospital, Breda, The Netherlands.

Correspondence : K.L.M. Koenraadt, Amphia, Molengracht 21, 4818 CK Breda, The Netherland.

Email : koenkoenraadt@gmail.com

- 2021, Acta Orthopædica Belgica.

Acta Orthopædica Belgica, Vol. 87 - 2 - 2021 
the fracture patients in comparison to OA patients $(2,3,5)$.Although patients with an acute femoral neck fracture were generally older, with a higher percentage of women, these factors could not fully explain the differences in outcome (1).

Many reasons have been suggested to explain the increased risk of failure after THA in fracture patients, including patient-related issues such as specific medical conditions (6) and increased age. ${ }^{5}$ Furthermore, risk of failure due to periprosthetic fracture may be gender related (6,7). As the main reasons for revision were dislocation and periprosthetic fractures $(2,3,5)$, technical issues may also influence the outcome. Choice of approach and head size, type of implant and method of fixation are all potential influential factors. The experience of the orthopedic surgeon in using a certain implant and approach is very relevant $(8,9)$. It is almost impossible for registry studies to take all these issues into account, therefore the outcome presented in registry studies may contain many confounding factors.

Registries have so far been unable to explain the difference in outcome between THA in OA patients and acute patients with a femoral neck fracture. To study if primary diagnosis alone is a reason for an inferior outcome in THA for acute femoral neck fracture, we conducted a single center cohort study in which patient-related issues are taken into consideration, and surgeon and implant related factors are minimized. In this setting we aimed to determine whether the primary diagnosis OA or femoral neck fracture is of importance relating to the prosthetic survival after THA. Secondarily, we considered whether patient or surgical factors were influential to the outcome of THA. We also tried to identify any reasons for failure between the groups.

\section{PATIENTS AND METHODS}

From March 2009 to September 2014, all primary THAs at our institution were analyzed. All THA data were collected in a local database. Additional information was gathered from electronic patient records. To ascertain correct survival data, the national population registry was checked for patient mortality and prosthesis data were verified from the nationwide arthroplasty registry. Patients with a minimum follow-up of 2 years were included.

In total 3412 patients had undergone a primary THA in this time period. We selected patients with a primary diagnosis of osteoarthritis and acute femoral neck fracture. We excluded THA for other primary diagnosis $(\mathrm{N}=278)$. To exclude influence of the type of implant in the current analysis, we included all patients with an Accolade TMZF stem (Stryker Orthopedics, Mahwah, USA) in combination with a Trident acetabular cup (Stryker Orthopedics, Mahwah, USA). This is the primary implant of choice at our institute. Both implants have an ODEP 10A* rating. We used a $36 \mathrm{~mm} \mathrm{CoCr}$ LFIT V40 head or Biolox Delta Ceramic V40 head and an X3 polyethylene insert (Stryker Orthopedics, Mahwah, USA) in all patients. Patients who had received another implant (cemented or hybrid THA or reconstructions with a modular implant) were excluded for the current analysis $(\mathrm{N}=308)$. Reasons for using a cemented or hybrid THA or primary THA with modular implants at our institute include patients with deformities that interfere with an adequate proximal press-fit on the femoral side, such as post-traumatic changes with osteosynthesis in situ, patients with earlier hip surgery (osteotomy), patients with metastasis and sizing or rotational issues in dysplastic patients. On the acetabular side it includes patients with large cysts or dysplastic acetabuli that require grafting with a cemented cup, post-traumatic changes with bone defects or patients with metastasis. These patients were treated with another implant and not taken into account in the current analysis. Additionally, we excluded a small cohort of patients operated with the direct anterior approach $(\mathrm{N}=44)$ to prevent a learning curve effect in these patients (10), similar to results from the national registry (11). In total 2782 primary THA were available for analysis. We made two research groups based on primary diagnosis for THA. One group of 2610 hips contained elective patients who had received a primary THA for osteoarthritis (OA Group), the other group of 172 hips consisted of acute patients with a femoral neck fracture (Fracture Group). In our institution, THA for acute femoral neck fracture is performed in patients with unrestricted ability to walk without walking aids 
Table I. - Demographic data from the patient groups and surgical approach

\begin{tabular}{|l|c|c|}
\hline & OA Group (N=2610) & Fracture Group (N=172) \\
\hline Age*(years) & 70.0 (SD 9.7) & $72.1($ SD 8.5) \\
\hline Gender (male \%) & 34.6 & 30.2 \\
\hline ASA* $^{*}$ & $1-16.4 \%$ & $1-11.0 \%$ \\
& $2-58.9 \%$ & $2-58.7 \%$ \\
& $3-24.6 \%$ & $3-29.7 \%$ \\
& $4-0.2 \%$ & $4-0.6 \%$ \\
\hline Approach* & Posterior $-95.9 \%$ & Posterior $-88.4 \%$ \\
& Anterolateral - 4.1\% & Anterolateral $-11.6 \%$ \\
\hline
\end{tabular}

* indicates a significant difference between the groups.

before the fracture, especially in patients under 80 years. In patients older than 80 years, there is a higher tendency to perform a hemiarthroplasty. All primary THAs were performed by the same group of orthopedic surgeons. Each surgeon used their own preferred hip approach (Table I) and all patients followed the same postoperative care protocol.

Demographic data included age, gender and ASA-grade, which are shown in Table I. In the Fracture Group 119 THAs (69\%) were performed on the day of admission, an additional 44 THAs $(25 \%)$ were carried out on the following day. In 9 THA (5\%) surgery was delayed for more than 24 hours. Normally distributed data are presented by mean (SD), otherwise using median (interquartile range).

Primary outcome was the difference in risk of revision for all reasons between the Fracture and the OA group. Therefore, the effect of primary diagnosis on the risk of revision was determined with a Cox regression analysis correcting for age, gender, ASAgrade and approach. We used the Kaplan-Meier survival analysis to determine the survival rates. Fisher's Exact tests were performed to analyze the different reasons for revision (dislocation, periprosthetic fracture, aseptic loosening, infection and other) between the OA Group and Fracture Group. All statistical analyses were performed with SPSS (v24, IBM Statistics).

\section{RESULTS}

In the Fracture Group, the patients were older, were operated more frequently using the anterolateral approach and had a higher ASA-grade in

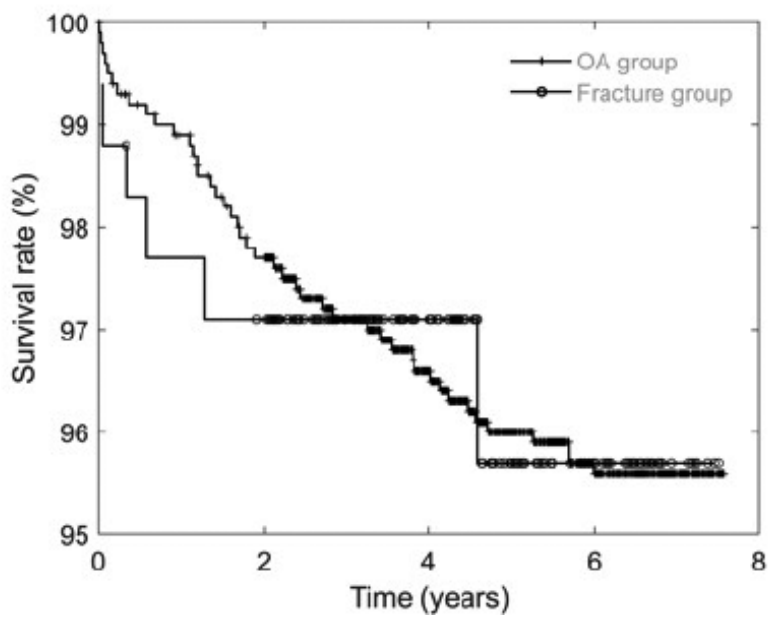

Figure 1. - Kaplan-Meier survival curve for the OA Group and Fracture Group.

comparison to the OA Group (Table I). The mean follow-up time was 4.6 years (range 2-7.6 years). In total 100/2782 hips (3.6\%) were revised during follow-up. There were 94 revisions in the OA Group (3.6\%) and 6 revisions in the Fracture Group (3.5\%). During follow-up, 7 (4.1\%) patients had died after the THA in the Fracture group and 89 (3.4\%) in the OA group, which was comparable $(\mathrm{p}=0.65)$. Cumulative survival of the THA at 5 years follow-up was $96.0 \%(95 \% \mathrm{CI}: 95.2-96.8)$ in the OA Group and $95.7 \%$ (95\% CI : $91.5-98.9)$ in the Fracture Group (Fig. 1). The risk of revision was comparable between the fracture and OA group $(\mathrm{HR}=1.04,95 \% \mathrm{CI}: 0.46-2.39)$.

Cox regression analysis revealed that the primary diagnosis (OA group vs. Fracture group) had no association with the risk of revision for any reason after THA $(p=0.92)$, nor did age $(p=0.82)$, ASA 
Table II. - Hazard ratios resulting from the Cox regression analysis

\begin{tabular}{|c|c|c|c|}
\hline $\begin{array}{c}\text { Diagnosis } \\
\text { OA }\end{array}$ & HR & $95 \%$ CI & p-value \\
\hline Fracture & 1.043 & $0.455-2.390$ & 0.92 \\
\hline $\begin{array}{c}\text { ASA } \\
\text { I }\end{array}$ & 1.008 & $0.514-1.977$ & 0.98 \\
\hline II & 1.080 & $0.670-1.741$ & 0.75 \\
\hline III-IV & 1 (Ref.) & & \\
\hline $\begin{array}{c}\text { Age } \\
<50\end{array}$ & 0.868 & $0.259-2.908$ & 0.82 \\
\hline 50-60 & 0.725 & $0.342-1.536$ & 0.40 \\
\hline $60-75$ & 0.846 & $0.549-1.305$ & 0.45 \\
\hline$>75$ & 1 (Ref.) & & \\
\hline $\begin{array}{c}\text { Gender } \\
\text { Male }\end{array}$ & 0.973 & $0.639-1.483$ & 0.90 \\
\hline Female & 1 (Ref.) & & \\
\hline $\begin{array}{c}\text { Surgical Approach } \\
\text { Posterolateral }\end{array}$ & 0.803 & $0.350-1.842$ & 0.60 \\
\hline Anterolateral & 1 (Ref.) & & \\
\hline
\end{tabular}

$(\mathrm{p}=0.94)$, gender $(\mathrm{p}=0.90)$ and surgical approach $(\mathrm{p}=0.60) \quad$ (Table II). We found no significant difference between the OA Group and Fracture Group in reasons for revision (Table III). Aseptic loosening was the main reason for revision. Revision for aseptic loosening was performed in 40 hips $(1.4 \%)$.

We found no difference in periprosthetic fracture as a reason for revision between the $\mathrm{OA}$ and Fracture Groups (Table III). Periprosthetic fracture occurred within 3 months with minor or no trauma after initial surgery in 14 cases $(0.5 \%)$. With regard to dislocation as the reason for revision, also no differences were ascertained between the two groups (Table III). Finally, also for the other reasons for revision no differences between the OA and Fracture
Groups were found (Table III). In 13 hips a revision procedure was performed for infection $(0.5 \%)$, including DAIR procedures with liner and head exchange. In 6 hips $(0.2 \%)$ revision was performed for other reasons. In total, there were 74 femoral stem revisions $(2.7 \%)$ and 30 acetabular component $(1.1 \%)$ revisions. In 16 cases both cup and stem were revised, in eleven cases the reason for revision was infection, in 3 cases aseptic loosening and in the remaining 2 cases dislocation was marked as the reason for revision. Stem survival with endpoint revision for any reason was $97.1 \%$ (95\% CI : 96.497.8) and cup survival $98.7 \%$ (95\% CI : 98.3-99.2) at 5 years follow-up in this cohort.

\section{DISCUSSION}

We performed a single center cohort study comparing the outcome of THA for femoral neck fracture with THA in OA patients. We compared the results of the same group of hip surgeons, using the same type of implant in both groups with an adequate ODEP-rating. We did not find a difference in survival rate at 5 years follow-up or a difference in reasons for failure between the two research groups. Therefore, THA was deemed equally effective in OA patients and fracture patients without a higher risk of dislocation or periprosthetic fracture in the fracture patients in this cohort.

In contrast to registry studies (1-4), we did not find a significant difference in survival between THA in elective OA and THA for acute femoral neck fracture. In this study, an adequate survival rate was found, which aligns with findings in the British National Joint Registry relating to use of this implant at 5 years follow-up (1). The discrepancy between the registries and our study with regard to the indication

Table III. - Revisions in the OA Group and Fracture Group during follow-up

\begin{tabular}{|l|c|c|c|}
\hline & OA Group: 94 (3.6\%) & $\begin{array}{c}\text { Fracture Group: 6 } \\
\mathbf{( 3 . 5 \% )}\end{array}$ & p-value \\
\hline Aseptic loosening & $37(1.4 \%)$ & $3(1.7 \%)$ & 0.73 \\
\hline Dislocation & $16(0.6 \%)$ & $2(1.2 \%)$ & 0.38 \\
\hline Periprosthetic fracture & $22(0.8 \%)$ & $1(0.6 \%)$ & 0.71 \\
\hline Infection & $13(0.5 \%)$ & 0 & 0.35 \\
\hline Other & $6(0.2 \%)$ & 0 & 0.50 \\
\hline
\end{tabular}


(OA or fracture) as a single parameter for difference in outcome might be the result of multiple causal factors. Many issues influence the outcome of THA and must be considered when interpreting the results of studies with heterogeneous patient populations. Dislocation and periprosthetic fracture were found more frequently as a reason for failure in THA in fracture patients in registry studies $(2,3,12)$. However, several factors may act as confounding factors in registry studies, although most effort is made to correct for as many factors as possible. In our study, all patient-related issues are well-known and can be fully corrected for. The influence of the individual surgeon in this study, and that of the chosen implant were minimized. This may be the reason for the difference in findings between this study and registry studies. Hence, when THA was performed with an implant with an adequate ODEPrating and by experienced hip surgeons, THA for acute femoral neck fracture did not influence the survival of the implant.

Results from different national hip arthroplasty registries revealed that revision for dislocation was increased in patients who had a THA for acute femoral neck fracture $(2,3)$. Many factors have been proposed as influential to the risk of dislocation. Dislocation of primary THA especially is related to femoral head size and the surgical approach $(3,11,13)$. In this study, we found a low risk of revision for dislocation at 5 years follow-up with no difference between the OA or fracture patients. In some studies, the posterior approach was associated with an increased risk of revision for dislocation $(3,11)$, although other studies show otherwise $(14,15)$. In patients with femoral neck fractures a higher dislocation rate was found both in THA (16), but also in hemiarthroplasty (17). Therefore, the primary diagnosis of femoral neck fracture alone would indeed seem a risk factor. However, in the study evaluating the dislocation rate of THA in patients with femoral neck fractures (16), the overall dislocation rate of $4.5 \%$ was much higher than in our study. This high dislocation rate was seen in THA performed with the posterior approach in combination with $22 \mathrm{~mm}$ heads and in which more than $50 \%$ of the cases the posterior capsule was not repaired. A dislocation rate as high as $13.6 \%$ was described in these patients (16). It is questionable whether the primary diagnosis or technical details in the approach were the main factor for the high dislocation rate. In a meta-analysis it was shown that leaving the capsule unrepaired increases the risk of dislocation 8-fold using the posterior approach (18). In a study from the Dutch Arthroplasty Register, the posterior approach in combination with a higher head size could effectively decrease the dislocation rate and was associated with a low rate of THA failure (11). Our study shows that use of the posterior approach by an experienced hip surgeon with adequate posterior repair in combination with a larger head size results in a low risk of revision for dislocation, also when THA was performed for femoral neck fracture. Therefore, in this setting the urge to follow the trend of increasing use of dual mobility cups in THA, with the accompanying additional costs, is lacking.

In literature, a higher risk of periprosthetic fracture was found in patients with THA for femoral neck fracture compared to THA for OA $(2,5)$. Periprosthetic fracture may be surgery related due to the type of implant or the procedure itself. In the current cohort most periprosthetic fractures occurred in elderly females with low energy trauma within 3 months after surgery. A minority of the patients had a fracture due to an adequate trauma. Overall at 5 years after THA, incidence rates for periprosthetic fractures of $0.8-1.0 \%$ were reported (19-21), which is comparable with $0.6-0.8 \%$ in the current study. Multiple risk factors for periprosthetic fracture after THA have been mentioned. In general, the risk for periprosthetic fracture is higher in females, with increasing age and if using an uncemented implant (7,22-24). Despite the fact that the fracture group consisted of older patients we did not find an increased risk of periprosthetic fracture between groups. With regard to the use of uncemented implants in our patients, generalization of outcome for cemented versus uncemented stems is very difficult. Large variability exists between certain types and shapes of implants (7,25-27), especially in combination with specific patient characteristics such as femoral shape or frail elderly $(5,20,28)$. In patients with hip arthroplasty for femoral neck fractures higher incidences of periprosthetic fractures 
were found for uncemented stems with rates of $15 \%$ at 2 years for the Zweymuller stem (29) and $7.4 \%$ at 5 years for the Corail stem (30). Incidence rates for periprosthetic fractures in patients with displaced femoral neck fractures with cemented stems were $1.3 \%$ at 2 years for the Exeter stem (29) and $0.9 \%$ at 5 years for the Spectron stem (30). The Accolade TMZF stem used in the current study is an anatomically shaped uncemented stem, without overloading the proximal femur and the finding in our study of $0.8 \%$ periprosthetic fractures after almost 5 years follow-up is in agreement with the literature for primary THA. The choice of implant especially, seems to influence the periprosthetic fracture rate rather than the primary diagnosis. Compared to incidences reported in the literature, low periprosthetic fracture rates can be achieved in patients with THA for femoral neck fracture also using an uncemented implant.

In the case of a hip fracture, early intervention (if patients fitness allows surgery) reduces pain, the incidence of pressure sores and the length of hospital stay. Although correctable medical conditions should be addressed, surgery should preferably be performed within 12-48 hours (31). In the current study most patients in the Fracture Group were operated within 24 hours. We used the same implant in our fracture patients as we normally use in our OA patients. These implants all have an ODEP $10 A^{*}$ rating. Both groups were treated by the same set of experienced orthopedic surgeons and had the same aftercare protocol. All these factors together resulted in equally good results after THA in both $\mathrm{OA}$ and acute femoral fracture patients.

One of the limitations of this study is the relatively small number of patients included. Although a large cohort of patients was investigated, it is not comparable to national joint registries. This also accounts since in the current analysis only the patients eligible for THA with the specific uncemented implant were included. However, in contrast to registry studies all specific patient data were known and the group was relatively homogenous, which resulted in less confounders influencing the research question.

In conclusion, the current study was not able to allocate the primary diagnosis femoral neck fracture as the sole reason for decreased outcome of THA when compared to OA patients. Standard use of an implant with an adequate ODEP rating, surgery undertaken by experienced hip surgeons, prompt surgical intervention and standardize aftercare resulted in a comparable outcome of THA in femoral neck fracture and OA patients and may be more important than the primary diagnosis.

\section{REFERENCES}

1. National Joint Registry : 14th Annual Report. 2017.

2. Gjertsen JE, Lie SA, Fevang JM, et al. Total hip replacement after femoral neck fractures in elderly patients: Results of 8,577 fractures reported to the Norwegian Arthroplasty Register. Acta Orthop. 2007 ; 78(4) : 491-7.

3. Hailer NP, Weiss RJ, Stark A, Kärrholm J. The risk of revision due to dislocation after total hip arthroplasty depends on surgical approach, femoral head size, sex, and primary diagnosis. Acta Orthop. 2012 ; 83(5) : 442-8.

4. Tarasevicius S, Cebatorius A, Valaviciene R, Stucinskas J, Leonas L, Robertsson O. First outcome results after total knee and hip replacement from the Lithuanian arthroplasty register. Med. $2014 ; 0(2): 87-91$.

5. Brodén C, Mukka S, Muren O, et al. High risk of early periprosthetic fractures after primary hip arthroplasty in elderly patients using a cemented, tapered, polished stem : An observational, prospective cohort study on 1,403 hips with 47 fractures after mean follow-up time of 4 years. Acta Orthop. $2015 ; 86(2)$ : 169-74.

6. Tsai C, Muo C, Hung C, Lin T, Wang T, Fong Y. Disorderrelated risk factors for revision total hip arthroplasty after hip hemiarthroplasty in displaced femoral neck fracture patients : a nationwide population-based cohort study. $J$ Orthop Surg Res. 2016 ; 1-9.

7. Thien TM, Chatziagorou G, Garellick G, et al. Periprosthetic femoral fracture within two years after total hip replacement : Analysis of 437,629 operations in the nordic arthroplasty register association database. J Bone Jt Surg Am. 2014 ; 96(19) : e167.

8. Manley M, Ong K, Lau E, Kurtz SM. Effect of volume on total hip arthroplasty revision rates in the United States Medicare population. J Bone Joint Surg Am. 2008 ; 90(11) : 2446-51.

9. Ravi B, Jenkinson R, Austin PC, et al. Relation between surgeon volume and risk of complications after total hip arthroplasty : Propensity score matched cohort study. BMJ. $2014 ; 348$ : g3284.

10. Spaans AJ, Hout J a a M Van Den, Bolder SBT. High complication rate in the early experience of minimally invasive total hip arthroplasty by the direct anterior approach. Acta Orthop. 2012 ; 83(4) : 342-6.

11. Zijlstra WP, De Hartog B, Van Steenbergen LN, Scheurs BW, Nelissen RGHH. Effect of femoral head size and 
surgical approach on risk of revision for dislocation after total hip arthroplasty : An analysis of 166,231 procedures in the Dutch Arthroplasty Register (LROI). Acta Orthop. 2017 ; 88(4) : 395-401.

12. Hailer NP, Lazarinis S, Mäkelä KT, et al. Hydroxyapatite coating does not improve uncemented stem survival after total hip arthroplasty! Acta Orthop. 2015 ; 86(1) : 18-25.

13. Conroy JL, Whitehouse SL, Graves SE, Pratt NL, Ryan P, Crawford RW. Risk Factors for Revision for Early Dislocation in Total Hip Arthroplasty. J Arthroplasty. $2008 ; 23(6): 867-72$.

14. Jolles B, Bogoch E. Posterior versus lateral surgical approach for total hip arthroplasty in adults with osteoarthritis. Cochrane Database Syst Rev. 2006 ; (3): Art. No. : CD003828.

15. Palan J, Beard DJ, Murray DW, Andrew JG, Nolan J. Which approach for total hip arthroplasty : Anterolateral or posterior? Clin Orthop Relat Res. 2009 ; 467(2) : 473-7.

16. Enocson A, Hedbeck CJ, Tidermark J, Pettersson H, Ponzer S, Lapidus LJ. Dislocation of total hip replacement in patients with fractures of the femoral neck A prospective cohort study of 713 consecutive hips. Acta Orthop. 2009 ; 80(2) : 184-9.

17. Enocson A, Tidermark J, Törnkvist H, Lapidus LJ. Dislocation of hemiarthroplasty after femoral neck fracture : Better outcome after the anterolateral approach in a prospective cohort study on 739 consecutive hips. Acta Orthop. $2008 ; 79(2): 211-7$.

18. Kwon MS, Kuskowski M, Mulhall KJ, Macaulay W, Brown TE, Saleh KJ. Does surgical approach affect total hip arthroplasty dislocation rates? Clin Orthop Relat Res. 2006 ; (447) : 34-8.

19. Cook RE, Jenkins PJ, Walmsley PJ, Patton JT, Robinson CM. Risk factors for periprosthetic fractures of the hip : A survivorship analysis. Clin Orthop Relat Res. 2008 ; 466(7) : 1652-6.

20. Meek RMD, Norwood T, Smith R, Brenkel IJ, Howie CR. The risk of peri-prosthetic fracture after primary and revision total hip and knee replacement. J Bone Jt Surg Br. 2011 ; 93-B : 96-101.

21. Berry DJ. Epidemiology : hip and knee. Orthop Clin North Am. $1999 ; 30(2): 183-90$.

22. Chammout G, Muren O, Laurencikas E, et al. More complications with uncemented than cemented femoral stems in total hip replacement for displaced femoral neck fractures in the elderly: A single-blinded, randomized controlled trial with 69 patients. Acta Orthop. 2017 ; 88(2) : 145-51.

23. Gjertsen JE, Lie SA, Vinje T, et al. More re-operations after uncemented than cemented hemiarthroplasty used in the treatment of displaced fractures of the femoral neck : An observational study of 11116 hemiarthroplasties from a national register. J Bone Jt Surg Br. 2012 ; 94-B : 1113-9.

24. Lindberg-Larsen M, Jørgensen CC, Solgaard S, Kjersgaard AG, Kehlet H. Increased risk of intraoperative and early postoperative periprosthetic femoral fracture with uncemented stems : 7,169 total hip arthroplasties from 8 Danish centers. Acta Orthop. 2017 ; 88(4) : 390-4.

25. Gromov K, Bersang A, Nielsen CS, Kallemose T, Husted H, Troelsen A. Risk factors for post-operative periprosthetic fractures following primary total hip arthroplasty with a proximally coated double-tapered cementless femoral component. Bone Joint J. 2017 ; 99-B : 451-7.

26. Van Eynde E, Hendrickx M, Scheerlinck T. Uncemented femoral stem design influences the occurrence rate of postoperative fractures after primary hip arthroplasty: A comparison of the Image ${ }^{\circledR}$ and Profile ${ }^{\circledR}$ stems. Acta Orthop Belg. 2010 ; 76(2) : 189-98.

27. Watts CD, Abdel MP, Lewallen DG, Berry DJ, Hanssen AD. Increased Risk of Periprosthetic Femur Fractures Associated With a Unique Cementless Stem Design. Clin Orthop Relat Res. 2015 ; 473(6) : 2045-53.

28. Mukka S, Mellner C, Knutsson B, Sayed-Noor A, Sköldenberg O. Substantially higher prevalence of postoperative periprosthetic fractures in octogenarians with hip fractures operated with a cemented, polished tapered stem rather than an anatomic stem. Acta Orthop. $2016 ; 87(3)$ : 257-61.

29. Taylor F, Wright M, Zhu M. Hemiarthroplasty of the Hip with and without Cement : A Randomized Clinical Trial. $J$ Bone Jt Surg Am. 2012 ; 94 : 577-83.

30. Langslet E, Frihagen F, Opland V, Madsen JE, Nordsletten L, Figved W. Cemented versus uncemented hemiarthroplasty for displaced femoral neck fractures: 5-year followup of a randomized trial. Clin Orthop Relat Res. 2014 ; 472(4) : 1291-9.

31. Lewis PM, Waddell JP. When is the ideal time to operate on a patient with a fracture of the hip? A review of the available literature. Bone Joint J. 2016 ; 98-B : 1573-81. 